\title{
Classification of ovarian cancer cell lines using transcriptional profiles defines the five major pathological subtypes
}

Barnes, B. M. ${ }^{1}$, Nelson, L. ${ }^{1}$, Tighe, A. ${ }^{1}$, Morgan, R. D. ${ }^{1}$, McGrail, J., and Taylor, S. S. ${ }^{1 *}$

1 Division of Cancer Sciences, Faculty of Biology, Medicine and Health, University of Manchester, Manchester Cancer Research Centre, 555 Wilmslow Road, Manchester M20 4GJ, United Kingdom.

* Lead Contact and Corresponding Author: stephen.taylor@manchester.ac.uk

Manuscript details:

Page numbers 17

Figures \& tables $\quad 3$

Supplemental information $\quad 1$ Figure and 2 Tables 


\section{Abstract}

Epithelial ovarian cancer $(E O C)$ is a heterogenous disease consisting of five major pathologically distinct subtypes: High-grade serous ovarian carcinoma (HGSOC), low-grade serous

4 (LGS), endometrioid, clear cell and mucinous carcinoma. Although HGSOC is the most prevalent

5 subtype, representing approximately $75 \%$ of cases, a 2013 landmark study from Domcke et al.,

6 found that many frequently used ovarian cancer cell lines were not genetically representative of

7 HGSOC tissue samples from The Cancer Genome Atlas. Although this work subsequently identified

8 several rarely used cell lines to be highly suitable as HGSOC models, cell line selection for ovarian

9 cancer research does not appear to have altered substantially in recent years. Here, we find that

10 application of non-negative matrix factorisation (NMF) to the transcriptional profiles of 45 commonly

11 used ovarian cancer cell lines exquisitely clusters them into five distinct classes, representative of

12 the five main subtypes of EOC. This methodology was in strong agreement with Domcke et al., in

13 identification of cell lines most representative of HGSOC. Furthermore, this robust classification of

14 cell lines, including some previously not annotated or miss-annotated in the literature, now informs

15 selection of the most appropriate models for all five pathological subtypes of ovarian cancer.

16 Furthermore, using machine learning algorithms trained using the classification of the current cell

17 lines, we are able provide a methodology for future classification of novel EOC cell lines. 


\section{Introduction}

Ovarian cancer is the most common cause of gynaecological-related cancer death in Europe and North America (Bray et al., 2018). Epithelial ovarian cancer (EOC), which accounts for $80 \%$ of all ovarian tumours, is now considered to be a heterogeneous disease consisting of five main histological subtypes characterised by different clinical and molecular features (Lheureux et al., 2019). High-grade serous ovarian carcinoma (HGSOC) is the most prevalent group, accounting for approximately $75 \%$ of cases, while the remaining $25 \%$ are made up of low-grade serous (LGS), endometrioid, clear cell and mucinous carcinoma (Kurman et al., 2014). Endometrioid and mucinous carcinoma are further sub-classified into well, moderately and poorly differentiated tumours (grade 1 to 3, respectively) (Kurman et al., 2014). Diagnosis of each subtype of EOC involves histological examination in combination with immunohistochemistry analysis, which is considered gold standard (Kurman et al., 2014).

Expansion of next generation sequencing has enabled closer inspection of the unique genomes of each subtype of EOC. HGSOC are characterised by near-ubiquitous TP53 mutation and genome-wide copy-number variation (CNV), with germline or somatic BRCA1/2 variants present in $\sim 20 \%$ of cases (Bell et al., 2011; Ciriello et al., 2013; Huang et al., 2018). LGS less frequently shows TP53 mutation, and instead variants in the MAPK signalling pathway are observed (e.g. KRAS, NRAS, BRAF) (Etemadmoghadam et al., 2017; Fernandez et al., 2019; Jones et al., 2012). Clear cell carcinomas and well-differentiated (i.e., grade 1) endometrioid carcinomas are commonly associated with endometriosis and $A R I D-1 A$ variants (Jones et al., 2010; Wiegand et al., 2010). Finally, mucinous ovarian carcinoma is associated with KRAS variants and ERBB2 amplifications (Cheasley et al., 2019).

Cancer cell lines are often used as model systems to study cancer; however, most were established many years ago and have either genetically drifted from the original patient cells and/or lack sufficient clinical data to allow robust tumour type classification. For example, much of ovarian cancer research has been based on the SKOV-3 cell line, however an in-depth analysis of copynumber changes, mutations and microarray-based mRNA expression profiles revealed that this cell line and others are actually atypical, bearing few hallmarks of the most common type of ovarian cancer, HGSOC, as defined by comparison with patient samples from The Cancer Genome Atlas (Bell et al., 2011; Domcke et al., 2013). Indeed, this analysis by Domcke et al. represented a landmark in the field, identifying a number of Cancer Cell Line Encyclopaedia (CCLE) cell lines that better reflect the genomic and mRNA expression landscapes of HGSOC.

This raises a key question: without directly associated clinical and/or histopathological annotation, how does one determine which of the subtypes any given cell line or patient biopsy reflects? Here we set out to address this question by asking whether it is possible to distinguish EOC subtypes based on molecular fingerprints, in particular one derived from RNA-sequencing (RNAseq). While the utility of RNAseq as a tool for developing prognostic biomarkers is still in its infancy, the 
technique is tried and tested, has the potential to provide a wealth of information by interrogating the expression levels of tens of thousands of genes and is gradually becoming more accessible and less costly. The challenge is in the distilling of robust signatures that correlate with specific phenotypes from these complex datasets.

One approach to reducing the complexity of RNAseq data is non-negative matrix factorisation (NMF), which has been utilised to reduce the dimensionality of transcriptional profiles from thousands of genes to a subset of important metagenes, concurrently providing meaningful class discovery (Brunet et al., 2004). Here, we apply NMF to the gene expression profiles of 45 EOC cell lines sequenced as part of the CCLE. We demonstrate the decomposition of this panel of EOC cell lines into five robust clusters that recapitulate the characteristics of the different pathological histotypes. In turn, this allows reclassification of several cell lines that were previously not annotated or possibly miss-annotated. Our results align well with the analysis by Domcke et al., which was based on CCLE's earlier microarray gene expression dataset. Our analysis further facilitates selection of cell lines appropriate for research of HGSOC, and in addition identifies cell lines representing the other four EOC subtypes. We also provide a methodology for future classification of novel cell lines using a K-nearest neighbour (KNN) classifier trained on the CCLE cell lines. 


\section{Results and Discussion}

\section{Most frequently utilised CCLE lines are unlikely to be representative of HGSOC}

The analyses by Domcke et al. represents an important milestone in the field, ranking 47 ovarian cancer cell lines according to their genetic and gene expression resemblance to HGSOC. In the intervening seven years, additional data has become available, in particular RNAseq data. We therefore set out to revisit this issue. Our aim was to determine whether the next generation of gene expression profiling clusters EOC cell lines into the different histotypes by NMF, and evaluate the ability of common machine learning algorithms, KNN, random forest and support vector machine (SVM), trained to identify the NMF-assigned class.

Firstly, we performed an extensive literature search to collate all annotations related to the 47 CCLE cell lines with site of origin indicated to be the ovary (with available RNAseq data). This identified 44 cell lines of EOC origin, eliminating 3 representing the non-epithelial Brenner and granulosa tumour types, and an engineered/immortalised cell line. Information gathered included reported histotype, specimen site, pre-biopsy treatment, the HGSOC likelihood score (as determined by Domcke et al.) and any other relevant information, for example, age and clinical course (Table S1). We also determined cell line usage in research by PubMed search (see Table S2 for search terms, including aliases for each cell line). Interestingly cell line selection has not substantially altered in recent years, despite publication of Domcke's landmark study in 2013. Seven cell lines (ranked by most highly used: SKOV-3, A2780, OVCAR-3, IGROV-1, CAOV-3, 59M and OVCAR-8) collectively constitute almost $90 \%$ of the total PubMed citations (Fig. 1). Of these 7 , only three received a 'HGSOC-likely' score in the analysis by Domcke et al. (OVCAR-3, CAOV-3 and 59M). Strikingly, seven cell lines scoring highly as 'HGSOC-likely', KURAMOCHI, OVSAHO, SNU-119, COV362, OVCAR-4, COV318 and JHOS-4, only constitute $1.07 \%$ of PubMed usages of the 44 EOC cell lines included in the CCLE. Furthermore, as late as 2019, SKOV-3 and A2780 remain the first and second most highly studied cell lines in ovarian cancer research, respectively, despite their purported unsuitability as HGSOC cell line models.

\section{Cancer cell lines cluster into classes representative of the five EOC histotypes}

Next we obtained from the European Nucleotide Archive the raw RNAseq files for the 44 EOC cell lines analysed by the recent CCLE project (Ghandi et al., 2019) and mapped reads to the GRCh38 human genome assembly with gene annotations from Gencode v32. The most important parameter to estimate in any clustering method is the optimum number of clusters $(k)$ for the data. The consensus matrix methodology by Monti et al. (2003) is frequently used in the evaluation of clustering, where the entries of the consensus map are coloured from 0 to 1 , reflecting the probability of clustering of two samples together across multiple runs of NMF (see Fig. S1 for consensus maps of all NMF models from $\mathrm{k}$ of 2 to 7 ). 
Many quality metrics have been proposed to assess the optimum value of k (Fig. 2A): briefly, Brunet et al. (2004) proposed the cophenetic correlation coefficient, Kim and Park (2007) proposed the dispersion coefficient, Rousseeuw et al. (1987) proposed the silhouette width. In each instance, the value of $k$ that results in maximum of the coefficient is chosen as optimum. Additionally, Hutchins et al (2008) utilised the variation of the residual sums of squares (RSS) between the original data and estimated data (not shown). The value of $k$ at which the plot of RSS for each value of $k$ shows an inflection point can be chosen as the optimum. Plotting these metrics for 2 to 10 clusters revealed that both two and five clusters fitted the dataset well (Fig. 1A). However, at a factorisation rank of two, no biologically interpretable clustering was apparent, with cell lines reported as individual subtypes split across the two clusters (Fig. S1a). We backwards annotated each cell line with the cluster assignment from the NMF run using 5 clusters, and performed consensus clustering on the result of the NMF run using just two clusters (not shown). There was no readily observable stratification of the five clusters, or combination thereof, with each of the five clusters split across the two clusters. We inferred, therefore, that there were no nested structures present within the data as $\mathrm{k}$ was increased from 2 to 5 , as was observed previously in the classification of leukaemia samples using NMF (Brunet et al., 2004). Brunet et al. found that at a factorisation rank of 2, ALL and AML samples clustered separately. As the factorisation rank was increased from 2 to 3 , the ALL cluster divided into the T-cell and B-cell distinctions. Thus, NMF has been reported to reveal hierarchical structure when it exists, without forcing such structure on the data (as other clustering models may), highlighting the strengths of NMF over other methods (Brunet et al., 2004).

In the CCLE EOC dataset, NMF together with consensus clustering gave strong evidence for a five-class split with clear block diagonal patterns and correspondingly high-quality metrics, with $k=5$ cophenetic and silhouette width scores second only to k=2 (Fig. 2A). However, the dispersion score was highest for $k=5$ (Fig. 2A), and the RSS curve shows an inflection point at $k=5$ (not shown), tying $\mathrm{k}=2$ and $\mathrm{k}=5$ as the optimum. We then examined the subtype assigned by the primary literature source for each cell line (where available; Table S1). Interestingly, this showed a clear overrepresentation of cell lines from a given subtype contained within each cluster, suggesting that the clusters identified by NMF are representative of the major EOC subtypes of ovarian cancer (Fig. 1B).

\section{High grade serous ovarian carcinoma}

We begin our discussion of the five clusters with the top left of the consensus map (Fig. 2B; dark purple). Of the cell lines in this cluster, 8 of 16 were assigned 'serous' in their primary literature annotation. Of the remaining 8 cell lines, 1 was reported as endometrioid (COV362) and the subtype of the remaining 7 was not specified in the literature. Given the putative identification of this cluster as representing HGSOC-derived cell lines, we wanted to align our results with the likelihood scores of these cell lines determined in the analysis by Domcke et al. (Fig. 1B; blue/green graduated track). 
In fact, all 16 cell lines that fall within this cluster were within the top 20 scoring cell lines in the previous analysis, providing remarkable confirmation of the methodology used here and by Domcke et al. for annotating cell lines as representative of HGSOC. Of the cell lines not placed into the HGSOC cluster, but ranked in the top 20 of Domcke et al., TYK-nu and 59M were designated 'likely HGS' and JHOM-2B and ES2 'possibly HGSOC'. We discuss these cell lines in the context of their assigned cluster in the relevant sections below. Therefore, clustering, confirmed several cell lines without specified subtype in their primary literature source, to represent good models of HGSOC, including KURAMOCHI, OVCAR-4, Caov-4, OAW28, Caov-3, ONCO-DG-1, and OVCAR-3. The cell lines OVSAHO, SNU-119, COV318, JHOS-4, JHOS-2, OVKATE, FU-OV-1 and SNU-8 retained their literature classification as 'HGSOC' in our analysis.

COV362 was initially annotated as endometrioid in the literature, however here we find it clusters with the cell lines representing HGSOC. This line has a TP53 mutation and a BRCA2 mutation, lesions characteristic of HGSOC, supporting the placement of COV362 as HGSOC. However, it should be noted that SNU8 and, to a lesser extent, COV362, show disparate clustering across 200 runs of NMF with random initialisation points. COV362 also clustered $25 \%$ of the time into cluster 3 (low grade serous), suggesting that it may share some characteristics of these cell lines. Importantly, it does not cluster in any of the NMF runs with other cell lines reported as endometrioid, further suggesting that this designation may be incorrect. SNU8 also clustered in approx. $42 \%$ of NMF runs with cluster 3 (low grade serous) and in 14\% with cluster 4 (mucinous)

\section{Clear cell}

In the next cluster (second from the left; green), there is an enrichment of cell lines which were defined as clear cell in their primary literature source. In fact, of the 10 cells lines, 6 were annotated as clear cell in the original publication, 2 were annotated as serous, 1 mixed and 1 was not specified. No cell lines annotated primarily as clear cell in the literature fell into any other cluster. The two samples previously annotated as serous were EFO21 and OAW42. Indeed, both of these cell lines received relatively low HGSOC likelihood scores in the analysis by Domcke et al., suggesting they are poor HGSOC models. Unlike almost all HGSOC, OAW42 has wild-type TP53. However, it does harbour two separate frameshift mutations within ARID1A, supporting its designation here as clear cell (Wiegand et al., 2010). Although EFO21 has mutated TP53, and no ARID1A mutation, these cells have amplification of PIK3CA, showing resultant mRNA expression levels within the 93rd percentile of CCLE cell lines. The most common mutations identified by sequencing of a 46 gene panel using pure clear cell samples included mutations in PIK3CA (50.0\%; 52 of 104 cases tested), TP53 (18.1\%; 19/105), and KRAS (12.4\%; 13/105) (Friedlander et al., 2016). Our analysis therefore also supports EFO21 classification as a clear cell line.

The most heavily used ovarian cancer cell line, SKOV-3, also falls within this cluster. Despite its extensive use, the primary literature source does not designate SKOV-3 to any particular subtype. 
Interestingly, SKOV-3 may actually be one of the most typical examples of clear cell as they harbour aberrations of three of the most commonly mutated proteins in clear cell ovarian cancer: PIK3CA, ARID1A and TP53. Therefore, designation here as clear cell is most likely an accurate representation of this cell line.

\section{Low grade serous}

In our analysis TYK-nu and 59M cluster together in cluster 3, which we believe to represent LGS. The CCLE/broad institute report TYK-nu as having a TP53 mutation, which molecular studies of LGS suggest are less common in this subtype (8\% in LGS versus $96 \%$ in HGSOC) (Bell et al., 2011; Singer et al., 2005). However, LGS is also characterized by activation of the mitogen-activated protein kinase (MAPK) pathway. Mutations affecting this pathway are seen in KRAS, NRAS and $B R A F$ genes, in addition to multiple alterations affecting other genes related to this pathway (Etemadmoghadam et al., 2017; Fernandez et al., 2019; Jones et al., 2012). In addition, copy number alterations and mutations affecting 61 MAPK-related genes were recently identified in 14 LGS cell lines (Fernandez et al., 2019). In this vein, TYK-nu have two mutations within NRAS, a member of the RAS/RAF pathway not included within Domcke's scoring schema. Furthermore, TYK$\mathrm{nu}$ is derived from a 38-year-old patient in line with reports that LGS affects women at a younger age than HGSOC, with a median age at diagnosis for LGS of between 43 and 47 years (Gershenson, 2016; Gershenson et al., 2015). 59M, while also harbouring a TP53 mutation, has three mutations in proteins in the MAPK pathway (Ghandi et al., 2019), and is therefore characteristic of LGS (previously annotated as endometroid). (Wilson et al., 1996)

The group of Coscia et al. used a proteomic signature to stratify putative HGSOC cell lines into three distinct groups (Coscia et al., 2016). Although the majority of cell lines with a high genetic fidelity to HGSOC were classified as group I and bore a more epithelial proteome, the two cell lines that clustered in group III with a more mesenchymal proteome were 59M and TYK-nu. While there was a striking concordance between the proteomic signature of group I cell lines and HGSOC patient samples, as well as cultured fallopian tube epithelial cells, group III cell lines resembled the signature of immortalized ovarian surface epithelial cells. Although the authors suggest that heterogeneity exists in the proteome of HGSOC based on disparate sites of origin (Coscia et al., 2016), it could be argued that these differences actually represent the differences between HGSOC and LGS-derived cell lines.

Collectively, this suggests TYK-nu and 59M form part of a cluster of 8 LGS cell lines (Fig. $1 \mathrm{~B}$; light purple). As LGS represents a fairly recent descriptor, it is difficult to infer this annotation from primary literature annotations of cells lines. Here we identify 4 cell lines, TYK-nu, HeyA8, ES2, and OVCAR8, which were previously unspecified in the literature, to be representative of LGS. In addition, JHOM-1 also clusters here, which was initially annotated as mucinous in its primary literature source. 


\section{Mucinous}

Of five cancer cell lines annotated in their primary reference as mucinous, four of them fall into cluster number 4 . These are MCAS, RMUG-S, COV644 and JHOM-2B. Of the cell lines determined to be in the top 20 of HGSOC likely cell lines by Domcke et al., JHOM-2B is reported in the literature as mucinous and our NMF also clusters it with the majority of other mucinous cell lines, suggesting its original classification is correct. In fact, Domcke et al. ranked JHOM-2B as 19th, close to the threshold for designation as 'possibly HGS'. Indeed, this cell line does harbour a TP53 mutation, which may disproportionately influence its standing in the analysis by Domcke et al. However, while TP53 mutations are almost ubiquitous in HGSOC ovarian cancer, around $16 \%$ of mucinous tumours show mutated TP53 (Schuijer \& Berns, 2003). The fifth cell line reported as mucinous in its original publication is JHOM-1, falls into the cluster we tentatively class as LGS (discussed previously).

The cell line OV-90 also clusters with the mucinous cell line, which originally was not designated a subtype in the original articles. In support of its mucinous designation, it harbours ERB2 amplification and BRAF mutation which have been demonstrated in mucinous ovarian cancer (Cheasley et al., 2019; Friedlander et al., 2015).

\section{Endometrioid}

Finally, the fifth cluster, designated endometroid, is constituted of two cell lines that were annotated as such in their primary reference, namely TOV112D and OVK18. Two other cell lines annotated as endometroid in their primary reference fall into cluster 3 (which we tentatively label as the LGS cluster; 59M) and cluster 1 (HGSOC cluster; COV362), and their suitability to fit these clusters has been discussed previously. Two further cell lines that cluster as endometroid here, A2780 and OC314, were not assigned a subtype in their primary literature source and are therefore newly annotated as potential models of endometroid ovarian cancer.

Lastly, EFO-27 also clusters within the endometroid cluster. Although this cell line was originally classified as serous in the literature, it received a poor HGS-likelihood score in the work by Domcke et al., giving initial evidence of its unsuitability as a HGSOC model cell line. EFO27 cells harbour a missense mutation in PPP2R1A, which has previously been found to be mutated in $12.2 \%$ (5/41) of endometrioid ovarian cancers, but not in 50 high-grade and 12 low-grade serous carcinomas (McConechy et al., 2011). More recent genetic screens of endometrioid ovarian cancer identified similar driver mutations to endometrial carcinoma, including PTEN, CTNNB1, PIK3CA, ARID1A, TP53, KMT2D, KMT2B and PIK3R1 (Pierson et al., 2020). Indeed, with the exception of CTNNB1, EFO-27 have mutations in all these genes (Ghandi et al., 2019). Therefore, the genetic similarities between EFO-27 and endometrioid ovarian cancer support it representing a better model of this type of ovarian cancer, than of HGSOC. However, it should be noted that this cell line has a 
poor silhouette score in our consensus map (Fig. 2B), clustering with other endometrioid cell lines $58 \%$ of the time, and with cluster 4 (the mucinous cluster) in the other NMF runs. Of the genetic lesions associated with mucinous ovarian cancer (Friedlander et al., 2015), EFO-27 harbours PTEN and PIK3CA. This cell line does not harbour KRAS mutation or ERBB2 amplification, however, which have been shown to be mutated in mucinous ovarian cancer (Cheasley et al., 2019).

\section{Evaluating machine learning algorithms to classify ovarian cancer subtypes}

We next sought to determine whether the NMF class given to each cell line could be used to train a machine learning model to predict the subtype of a 'hold-out' set. Genes whose expression levels were characteristic for each cluster were extracted with each cluster containing between 23 and 82 such metagenes. The largest number of metagenes was associated with the putative HGSOC cluster (82), followed by, endometrioid (40), LGS (35), mucinous (28) and clear cell (23) (Fig. 3A). We next evaluated the classification potential of several common machine learning algorithms: KNN, random forests and SVM. The 45 cell lines were randomly partitioned into four groups, such that each group had an even representation of cell lines from each subtype. Then, each model was trained to each successive set of 3 groups, and model performance tested on the omitted group. This meant that each sample had an opportunity to be both trained and tested on. The per-subtype specificity and sensitivity metrics were compared across KNN, random forest and SVM algorithms (Fig. 3B). As can be seen, all models predicted the HGSOC subtype well, achieving balanced accuracy scores of 1 (KNN), 0.935275 (RF) and 0.984375 (SVM) for this class. This presumably reflects the larger number of samples labelled HGSOC and the number of metagenes present to predict this subtype versus the others. Therefore, additional samples representative of non-HGSOC ovarian cancer would greatly aid the training of a classifier. This is especially true in the case of endometrioid ovarian cancer cell lines, which was represented by only 4 of the 44 cell lines analysed in this study. Nevertheless, the overall kappa values achieved for each model was 0.918 (KNN), 0.78905 (RF) and 0.878 (SVM). This suggests that NMF coupled with KNN may be a powerful tool for ovarian cancer cell line subtype classification. 


\section{Conclusion}

The EOC subtype from which commonly used ovarian cancer cell lines were derived has remained a controversial topic for many years (Anglesio et al., 2013; Beaufort et al., 2014; Coscia et al., 2016; Domcke et al., 2013). We sought to determine whether recently released RNAseq data from the CCLE could shed light on this subject. Previous studies have sought to define an immunohistochemical, genetic or combinatorial panel, and determine the suitability of cells to fit this mould. Here we have not imposed any prior knowledge or structure onto the data, instead opting to use NMF, a clustering algorithm that has been used not only in gene expression studies, but other pattern-recognition problems such as facial recognition and deciphering the meaning of words (Brunet et al., 2004; Lee \& Seung, 1999). Our NMF clustering allowed cell lines to cluster with others that they most closely resembled at the transcriptional level, revealing novel subtype classifications for some cell lines. Inclusion of additional cell lines would improve the predictive utility of our machine-learning based classifier, especially subtypes that are underrepresented in the CCLE dataset, namely endometrioid and mucinous. Future work, therefore, could relate to the integration of multiple different sources of transcriptional profiles. Additionally, datasets containing patientderived cell lines could be utilised to further evaluate the performance of any classifier, including the recently published living ovarian biobank and others (Fernandez et al., 2019; Nelson et al., 2020). 


\section{Materials and Methods}

\section{Literature search}

We performed an extensive PubMed literature search to determine the usage of CCLE ovarian cancer cell lines. The list of search queries used is supplied in table S2, demonstrating the different aliases used for the different cell lines. It should be noted that these search queries only count the number of articles where the cell line name was specified in the title and/or abstract, therefore missing some articles that only specify within article the cell lines used. This will be especially true for larger studies that utilize many of these cell lines where it is not possible to list them in an abstract.

\section{RNAseq data}

Forty five cell lines representative of the major ovarian cancer subtypes analysed by RNAsequencing as part of the Cancer Cell Line Encyclopedia (CCLE) project (Ghandi et al., 2019) were identified (table S1). Raw sequence files in FASTQ format were obtained from the European Nucleotide Archive (ENA; http://www.ebi.ac.uk/ena/). STAR (v2.7.2a) (Dobin et al., 2013) was used to map reads to the GRCh38 human genome assembly with gene annotations from Gencode v32. The number of reads per gene were counted using --quantMode GeneCounts within the STAR command.

\section{Non-negative matrix factorisation}

Data analyses in R was performed using v3.6.2 and in Bioconductor v3.10. The DESeq2 (v1.26.0) (Love et al., 2014) package was used to apply a variance stabilizing transformation to the assembled read count matrix. Transcripts with a median absolute deviation $\geq 1.5$ were selected, and this list of 6,796 genes was used as input for clustering analysis using the NMF package (Gaujoux \& Seoighe, 2010). To estimate the factorisation rank (k), NMF was performed for a $k$ of 2 to 10 using 50 random initiations. Quality measures were computed for each factorisation rank, including the cophenetic coefficients, silhouette and RSS. Inspection of the computed quality metrics revealed 5 clusters fitted the data. Next, 200 iterative runs of NMF were performed from a fixed random initial condition with a $\mathrm{k}$ value of 5 . Using annotations given in the primary literature source for each cell line (table S1), we inferred the likely ovarian cancer histotype of each cluster. Gene scoring schema was applied to extract genes characteristic of the five identified clusters (Kim \& Park, 2007). Metagene lists were combined, and this was used as input for machine learning algorithms.

\section{Machine Learning Algorithms for Classification}

A plethora of classification algorithms have become available. Here, we explore the utility of three common classification algorithms: KNN, RF and SVM. We used the R package caret (v6.086) for model training and evaluation. The specific modules used were base::knn, randomForest (v4.6-14) and kernlab (v0.9-29), respectively. The cell lines with their subtype classifications 
outputted from our NMF analysis were partitioned into 4 random subsets, such that each set contained approximately equal proportions of each subtype. Models were trained using each combination of partitions, leaving one group out for testing of model performance in each instance. Metrics compared between models were the per-class (ability to predict each subtype, e.g. HGSOC, LGS etc.) sensitivity, specificity and balanced accuracy calculations. Overall model performance was compared using Cohen's kappa, which compares observed accuracy with the expected accuracy (subtypes predicted by a random classifier).

\section{K-nearest neighbours}

K-nearest neighbours is a non-parametric method proposed by Thomas Cover used for classification. A cell line within the held-out test set is classified by majority vote of its k-neighbours from the training set (although no explicit training step is required). $\mathrm{K}$ is typically a small positive integer, and usually of an odd number to avoid 'tied' decisions. A large k reduces the impact of variance caused by random error. However, this may miss the small but important patterns within the data (Zhang, 2016).

\section{Random Forrest}

Random forest is a learning method for classification, regression and other tasks. The forest is built from the construction of many different decision trees at training time. The power of the algorithm stems from the low-correlation between decision trees, which may cancel out the individual errors of any one tree. Each tree decides the subtype of a test-set cell line and the majority vote becomes the model's prediction. While some trees may be wrong, many other trees will be right, so as a group the trees are able to provide a more powerful prediction.

\section{SVM}

Support vector machine is a supervised machine learning algorithm that can be employed for both classification and regression purposes. SVM works by finding the decision boundary (the "hyperplane") that separates the classes of the supplied data, in our case the different subtypes of EOC. During training, the margins of the hyperplanes are maximised, while the cell lines remain on the correct side of the subtype boundaries. Intuitively, when the subtype of the test is predicted, we can be more confident that the prediction is correct if the cell lines lies further from the boundaries. Likewise, doubt is cast on the prediction of a cell line that sits close to the boundaries.

\section{Genetic background and copy number variation of CCLE cell lines}

The genetic background of the CCLE cell lines is extensively referred to throughout this manuscript. We direct the reader to the mutation and copy number variation datasets generated by this project. The datasets were originally presented in Ghandi et al (2019) and recommend the use of the cBioPortal for Cancer Genomics (https://www.cbioportal.org/) that enables interactive exploration of multidimensional cancer genomics data sets (Cerami et al., 2012; Gao et al., 2013). 


\section{Acknowledgments}

We thank the members of the Taylor lab for advice and comments on the manuscript. The research was funded by a Cancer Research UK Programme Grant to S.S.T (C1422/A19842) and the Cancer Research UK Centre Award (C5759/A25254).

\section{Author contributions}

Methodology, Investigation, Validation and Formal Analysis, B.B., L.N., A.T. and R.D.M.; Conceptualisation, B.B. and R.D.M; Writing, B.B., R.D.M., J.M. and S.S.T.; Funding and Supervision 378 S.S.T.

\section{Declaration of interests}

381 The authors declare no competing interests. 


\section{References}

Anglesio, M. S., Wiegand, K. C., Melnyk, N., Chow, C., Salamanca, C., Prentice, L. M., Senz, J., Yang, W., Spillman, M. A., Cochrane, D. R., Shumansky, K., Shah, S. P., Kalloger, S. E., \& Huntsman, D. G. (2013). Type-Specific Cell Line Models for Type-Specific Ovarian Cancer Research. PLoS ONE, 8(9). https://doi.org/10.1371/journal.pone.0072162

Beaufort, C. M., Helmijr, J. C. A., Piskorz, A. M., Hoogstraat, M., Ruigrok-Ritstier, K., Besselink, N., Murtaza, M., Van IJcken, W. F. J., Heine, A. A. J., Smid, M., Koudijs, M. J., Brenton, J. D., Berns, E. M. J. J., \& Helleman, J. (2014). Ovarian cancer cell line panel (OCCP): Clinical importance of in vitro morphological subtypes. PLOS ONE. https://doi.org/10.1371/journal.pone.0103988

Bell, D., Berchuck, A., Birrer, M., Chien, J., Cramer, D. W., Dao, F., Dhir, R., Disaia, P., Gabra, H., Glenn, P., Godwin, A. K., Gross, J., Hartmann, L., Huang, M., Huntsman, D. G., lacocca, M., Imielinski, M., Kalloger, S., Karlan, B. Y., ... Thomson, E. (2011). Integrated genomic analyses of ovarian carcinoma. Nature. https://doi.org/10.1038/nature10166

Bray, F., Ferlay, J., Soerjomataram, I., Siegel, R. L., Torre, L. A., \& Jemal, A. (2018). Global cancer statistics 2018: GLOBOCAN estimates of incidence and mortality worldwide for 36 cancers in 185 countries. CA: A Cancer Journal for Clinicians. https://doi.org/10.3322/caac.21492

Brunet, J. P., Tamayo, P., Golub, T. R., \& Mesirov, J. P. (2004). Metagenes and molecular pattern discovery using matrix factorization. Proceedings of the National Academy of Sciences of the United States of America, 101(12), 4164-4169. https://doi.org/10.1073/pnas.0308531101

Cerami, E., Gao, J., Dogrusoz, U., Gross, B. E., Sumer, S. O., Aksoy, B. A., Jacobsen, A., Byrne, C. J., Heuer, M. L., Larsson, E., Antipin, Y., Reva, B., Goldberg, A. P., Sander, C., \& Schultz, N. (2012). The cBio Cancer Genomics Portal: An open platform for exploring multidimensional cancer genomics data. Cancer Discovery. https://doi.org/10.1158/2159-8290.CD-12-0095

Cheasley, D., Wakefield, M. J., Ryland, G. L., Allan, P. E., Alsop, K., Amarasinghe, K. C., Ananda, S., Anglesio, M. S., Au-Yeung, G., Böhm, M., Bowtell, D. D. L., Brand, A., Chenevix-Trench, G., Christie, M., Chiew, Y. E., Churchman, M., DeFazio, A., Demeo, R., Dudley, R., ... Gorringe, K. L. (2019). The molecular origin and taxonomy of mucinous ovarian carcinoma. Nature Communications. https://doi.org/10.1038/s41467-019-11862-x

Ciriello, G., Miller, M. L., Aksoy, B. A., Senbabaoglu, Y., Schultz, N., \& Sander, C. (2013). Emerging landscape of oncogenic signatures across human cancers. Nature Genetics. https://doi.org/10.1038/ng.2762

Coscia, F., Watters, K. M., Curtis, M., Eckert, M. A., Chiang, C. Y., Tyanova, S., Montag, A., Lastra, R. R., Lengyel, E., \& Mann, M. (2016). Integrative proteomic profiling of ovarian cancer cell lines reveals precursor cell associated proteins and functional status. Nature Communications. https://doi.org/10.1038/ncomms12645

Dobin, A., Davis, C. A., Schlesinger, F., Drenkow, J., Zaleski, C., Jha, S., Batut, P., Chaisson, M., \& Gingeras, T. R. (2013). STAR: Ultrafast universal RNA-seq aligner. Bioinformatics, 29(1), 15-21. https://doi.org/10.1093/bioinformatics/bts635

Domcke, S., Sinha, R., Levine, D. A., Sander, C., \& Schultz, N. (2013). Evaluating cell lines as tumour models by comparison of genomic profiles. Nature Communications. https://doi.org/10.1038/ncomms3126

Etemadmoghadam, D., Azar, W. J., Lei, Y., Moujaber, T., Garsed, D. W., Kennedy, C. J., Fereday, S., Mitchell, C., Chiew, Y. E., Hendley, J., Sharma, R., Harnett, P. R., Li, J., Christie, E. L., Patch, A. M., George, J., Au-Yeung, G., Arnau, G. M., Holloway, T. P., ... DeFazio, A. (2017). EIF1AX and NRAS mutations co-occur and cooperate in low-grade serous ovarian carcinomas. Cancer Research. https://doi.org/10.1158/0008-5472.CAN-16-2224

Fernandez, M. L., Dawson, A., Hoenisch, J., Kim, H., Bamford, S., Salamanca, C., DiMattia, G., Shepherd, T., Cremona, M., Hennessy, B., Anderson, S., Volik, S., Collins, C. C., Huntsman, 
D. G., \& Carey, M. S. (2019). Markers of MEK inhibitor resistance in low-grade serous ovarian cancer: EGFR is a potential therapeutic target. Cancer Cell International.

https://doi.org/10.1186/s12935-019-0725-1

Friedlander, M. L., Russell, K., Millis, S., Gatalica, Z., Bender, R., \& Voss, A. (2016). Molecular profiling of clear cell ovarian cancers: Identifying potential treatment targets for clinical trials. International Journal of Gynecological Cancer, 26(4), 648-654. https://doi.org/10.1097//GC.0000000000000677

Friedlander, M., Russell, K., Millis, S. Z., Gatalica, Z., \& Voss, A. (2015). Molecular profiling of mucinous epithelial ovarian carcinomas (mEOC): Opportunities for clinical trials. Journal of Clinical Oncology, 33(15_suppl), 5540-5540. https://doi.org/10.1200/jco.2015.33.15_suppl.5540

Gao, J., Aksoy, B. A., Dogrusoz, U., Dresdner, G., Gross, B., Sumer, S. O., Sun, Y., Jacobsen, A., Sinha, R., Larsson, E., Cerami, E., Sander, C., \& Schultz, N. (2013). Integrative analysis of complex cancer genomics and clinical profiles using the cBioPortal. Science Signaling. https://doi.org/10.1126/scisignal.2004088

Gaujoux, R., \& Seoighe, C. (2010). A flexible R package for nonnegative matrix factorization. BMC Bioinformatics, 11, 367. https://doi.org/10.1186/1471-2105-11-367

Gershenson, D. M. (2016). Low-grade serous carcinoma of the ovary or peritoneum. Annals of Oncology. https://doi.org/10.1093/annonc/mdw085

Gershenson, D. M., Bodurka, D. C., Lu, K. H., Nathan, L. C., Milojevic, L., Wong, K. K., Malpica, A., \& Sun, C. C. (2015). Impact of age and primary disease site on outcome in women with low-grade serous carcinoma of the ovary or peritoneum: Results of a large single-institution registry of a rare tumor. Journal of Clinical Oncology. https://doi.org/10.1200/JCO.2015.61.0873

Ghandi, M., Huang, F. W., Jané-Valbuena, J., Kryukov, G. V., Lo, C. C., McDonald, E. R., Barretina, J., Gelfand, E. T., Bielski, C. M., Li, H., Hu, K., Andreev-Drakhlin, A. Y., Kim, J., Hess, J. M., Haas, B. J., Aguet, F., Weir, B. A., Rothberg, M. V., Paolella, B. R., ... Sellers, W. R. (2019). Next-generation characterization of the Cancer Cell Line Encyclopedia. Nature. https://doi.org/10.1038/s41586-019-1186-3

Huang, K. lin, Mashl, R. J., Wu, Y., Ritter, D. I., Wang, J., Oh, C., Paczkowska, M., Reynolds, S., Wyczalkowski, M. A., Oak, N., Scott, A. D., Krassowski, M., Cherniack, A. D., Houlahan, K. E., Jayasinghe, R., Wang, L. B., Zhou, D. C., Liu, D., Cao, S., ... Ding, L. (2018). Pathogenic Germline Variants in 10,389 Adult Cancers. Cell. https://doi.org/10.1016/j.cell.2018.03.039

Hutchins, L. N., Murphy, S. M., Singh, P., \& Graber, J. H. (2008). Position-dependent motif characterization using non-negative matrix factorization. Bioinformatics. https://doi.org/10.1093/bioinformatics/btn526

Jones, S., Wang, T. L., Kurman, R. J., Nakayama, K., Velculescu, V. E., Vogelstein, B., Kinzler, K. W., Papadopoulos, N., \& Shih, I. M. (2012). Low-grade serous carcinomas of the ovary contain very few point mutations. Journal of Pathology. https://doi.org/10.1002/path.3967

Jones, S., Wang, T. L., Shih, I. M., Mao, T. L., Nakayama, K., Roden, R., Glas, R., Slamon, D., Diaz, L. A., Vogelstein, B., Kinzler, K. W., Velculescu, V. E., \& Papadopoulos, N. (2010). Frequent mutations of chromatin remodeling gene ARID1A in ovarian clear cell carcinoma. Science. https://doi.org/10.1126/science.1196333

Kim, H., \& Park, H. (2007). Sparse non-negative matrix factorizations via alternating nonnegativity-constrained least squares for microarray data analysis. Bioinformatics. https://doi.org/10.1093/bioinformatics/btm134

Kurman, R. J., Carcangiu, M. L., Harrington, C. S., \& Young, R. H. (2014). WHO classification of tumours of female reproductive organs. IARC Press (Lyon).

Lee, D. D., \& Seung, H. S. (1999). Learning the parts of objects by non-negative matrix factorization. Nature, 401(6755), 788-791. https://doi.org/10.1038/44565 
Lheureux, S., Gourley, C., Vergote, I., \& Oza, A. M. (2019). Epithelial ovarian cancer. In The Lancet. https://doi.org/10.1016/S0140-6736(18)32552-2

Love, M. I., Huber, W., \& Anders, S. (2014). Moderated estimation of fold change and dispersion for RNA-seq data with DESeq2. Genome Biology, 15(12). https://doi.org/10.1186/s13059-0140550-8

McConechy, M. K., Anglesio, M. S., Kalloger, S. E., Yang, W., Senz, J., Chow, C., HeraviMoussavi, A., Morin, G. B., Mes-Masson, A. M., Bowtell, D., Chenevix-Trench, G., DeFazio, A., Gertig, D., Green, A., Webb, P., Carey, M. S., McAlpine, J. N., Kwon, J. S., Prentice, L. M., ... Huntsman, D. G. (2011). Subtype-specific mutation of PPP2R1A in endometrial and ovarian carcinomas. Journal of Pathology, 223(5), 567-573. https://doi.org/10.1002/path.2848

Monti, S., Tamayo, P., Mesirov, J., \& Golub, T. (2003). Consensus clustering: A resampling-based method for class discovery and visualization of gene expression microarray data. Machine Learning. https://doi.org/10.1023/A:1023949509487

Nelson, L., Tighe, A., Golder, A., Littler, S., Bakker, B., Moralli, D., Murtuza Baker, S., Donaldson, I. J., Spierings, D. C. J., Wardenaar, R., Neale, B., Burghel, G. J., Winter-Roach, B., Edmondson, R., Clamp, A. R., Jayson, G. C., Desai, S., Green, C. M., Hayes, A., ... Taylor, S. S. (2020). A living biobank of ovarian cancer ex vivo models reveals profound mitotic heterogeneity. Nature Communications. https://doi.org/10.1038/s41467-020-14551-2

Pierson, W. E., Peters, P. N., Chang, M. T., Chen, L. may, Quigley, D. A., Ashworth, A., \& Chapman, J. S. (2020). An integrated molecular profile of endometrioid ovarian cancer. Gynecologic Oncology, 157(1), 55-61. https://doi.org/10.1016/j.ygyno.2020.02.011

Rousseeuw, P. J. (1987). Silhouettes: A graphical aid to the interpretation and validation of cluster analysis. Journal of Computational and Applied Mathematics. https://doi.org/10.1016/03770427(87)90125-7

Schuijer, M., \& Berns, E. M. J. J. (2003). TP53 and ovarian cancer. In Human Mutation (Vol. 21, Issue 3, pp. 285-291). Hum Mutat. https://doi.org/10.1002/humu.10181

Singer, G., Stöhr, R., Cope, L., Dehari, R., Hartmann, A., Cao, D. F., Wang, T. L., Kurman, R. J., \& Shih, I. M. (2005). Patterns of p53 mutations separate ovarian serous borderline tumors and low- and high-grade carcinomas and provide support for a new model of ovarian carcinogenesis: A mutational analysis with immunohistochemical correlation. American Journal of Surgical Pathology. https://doi.org/10.1097/01.pas.0000146025.91953.8d

Wiegand, K. C., Shah, S. P., Al-Agha, O. M., Zhao, Y., Tse, K., Zeng, T., Senz, J., McConechy, M. K., Anglesio, M. S., Kalloger, S. E., Yang, W., Heravi-Moussavi, A., Giuliany, R., Chow, C., Fee, J., Zayed, A., Prentice, L., Melnyk, N., Turashvili, G., ... Huntsman, D. G. (2010). ARID1A mutations in endometriosis-associated ovarian carcinomas. New England Journal of Medicine. https://doi.org/10.1056/NEJMoa1008433

Wilson, A. P., Dent, M., Pejovic, T., Hubbold, L., \& Radford, H. (1996). Characterisation of seven human ovarian tumour cell lines. British Journal of Cancer, 74(5), 722-727. https://doi.org/10.1038/bjc.1996.428

Zhang, Z. (2016). Introduction to machine learning: K-nearest neighbors. Annals of Translational Medicine, 4(11). https://doi.org/10.21037/atm.2016.03.37 


\section{Figure legends}

Figure 1. Cell line usage based on PubMed citations. Top, total number of PubMed usages of each of the epithelial ovarian cancer cell lines for which RNAseq data is available within the CCLE. Bottom, HGSOC-likelihood scores as determined by Domcke et al. analysis of ovarian cancer cell lines correlated with The Cancer Genome Atlas HGSOC patient samples. Cell lines are separated along the $\mathrm{x}$-axis based on the year of their first usage. Cell lines are coloured by the subtype of epithelial ovarian cancer reported in their primary literature source. Green, clear cell; red, endometrioid; orange, mucinous; purple, serous; dark grey, mixed; light grey, not specified (NS).

Figure 2. Ovarian cancer cell lines can be divided into five clusters that recapitulate the histological subtypes based on transcriptional profiles. (A) Selected quality metrics describing the performance of non-negative matrix factorisation for 2 to 10 clusters. From left, the cophenetic correlation, dispersion and silhouette coefficients. Colours indicate the type of measure plotted. (B) Consensus map showing cell line clustering for 200 iterative runs of NMF using 5 clusters. The blocks of the consensus map are coloured by the probability of two samples clustering together, where red, 1; white, 0.5 and blue, 0 . The annotation track atop the heatmap indicates (top) the HGSOC-likelihood score of a cell line determined by Domcke et al. Where darker shades represent a higher score. The pure white blocks indicate the cell line was not included in this analysis. Middle track, the ovarian cancer subtype provided in the cell line's original literature source where green, clear cell; red, endometrioid; orange, mucinous; purple, serous; dark grey, mixed; light grey, not specified (NS). Bottom track, the consensus cluster assignment across 200 NMF runs where dark purple, cluster 1; green, cluster 2; light purple, cluster 3; orange, cluster 4 and red, cluster 5 .

Figure 3. A k-nearest neighbour classifies accurately predicts subtype of ovarian cancer cell lines. (A) Metagenes for which high expression is informative of each cluster were extracted using gene scoring scheme as per Kim and Park (2005). Colours represent the strength of the association between that gene and the cluster, where red, 1 and white, 0 . The track above the heatmap indicates cluster number, as per Fig. 2, where dark purple, cluster 1; green, cluster 2; light purple, cluster 3; orange, cluster 4 and red, cluster 5. (B) Evaluation of three machine learning algorithms for ovarian cancer cell line subtype classification, k-nearest neighbour (KNN), random forest (RF) and support vector machine (SVM). Cell lines were designated the subtype indicated by NMF clustering, and partitioned into 4 subsets. Three subsets were used to train each of the machine learning algorithms, with the fourth set held out as a test set. The four subsets were rotated such that each sample had the opportunity to be trained and tested upon. The average perclass sensitivity and specificity scores across the four tested sets is shown where dark purple, HGSOC; green, clear cell; light purple, LGS; orange, mucinous and red, endometrioid.

Figure $\mathbf{S 1}$ consensus cluster maps for NMF at different values of $k$. (A-F) consensus cluster maps (in order of increasing $\mathrm{k}$ ) from 2 to 7 clusters. The blocks of the consensus map are coloured by the probability of two samples clustering together, where red, 1 ; white, 0.5 and blue, 0 . The annotation tracks atop the heatmap indicate the ovarian cancer subtype provided in the cell line's original literature source where green, clear cell; red, endometrioid; orange, mucinous; purple, serous; dark grey, mixed; light grey, not specified (NS). Middle track, the consensus cluster assignment across 50 NMF runs. The cluster numbers and the colours assigned are shown in the legends to the right of each of the heatmaps. Bottom track, silhouette width for each sample pair where dark green indicates a silhouette width of 1 (perfect clustering). 


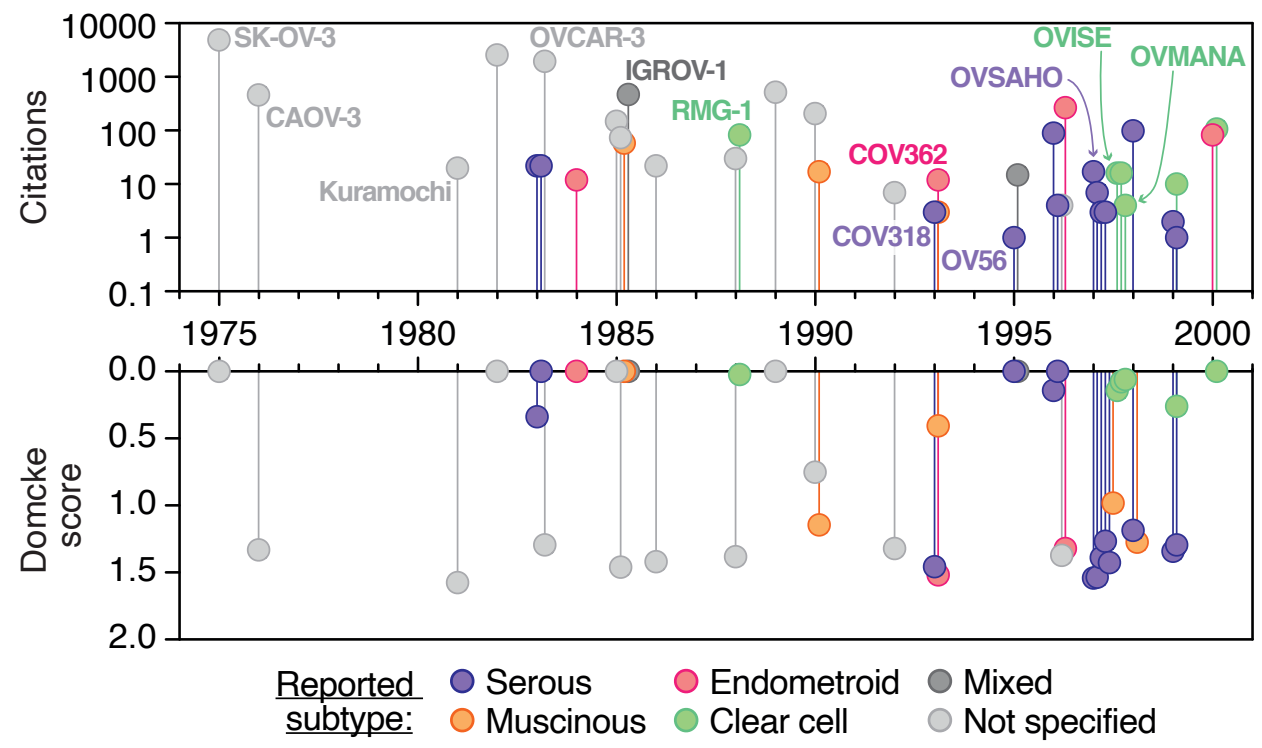

Figure 1 
bioRxiv preprint doi: https://doi.org/10.1101/2020.07.14.202457; this version posted July 15, 2020. The copyright holder for this preprint (which was not certified by peer review) is the author/funder. All rights reserved. No reuse allowed without permission.

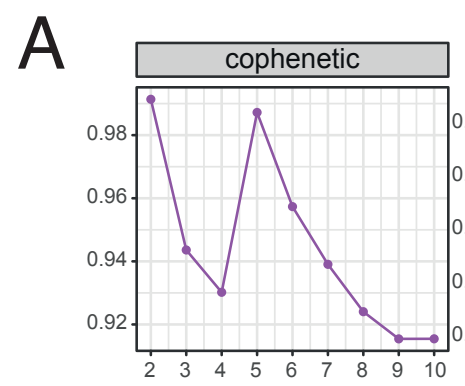

Factorization rank
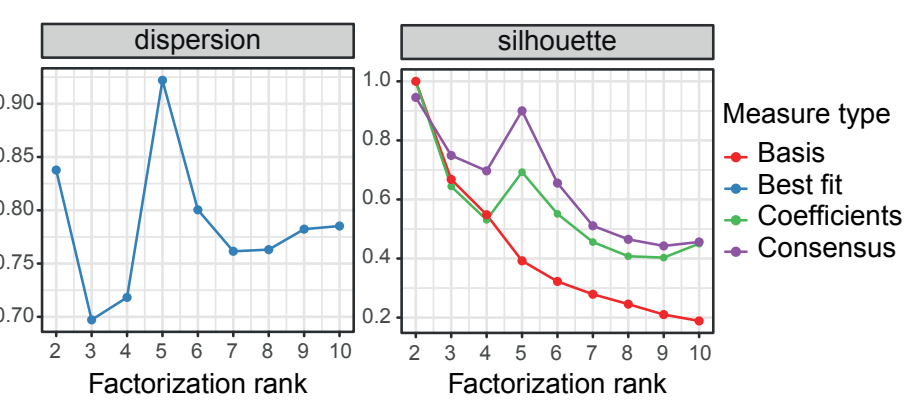

B

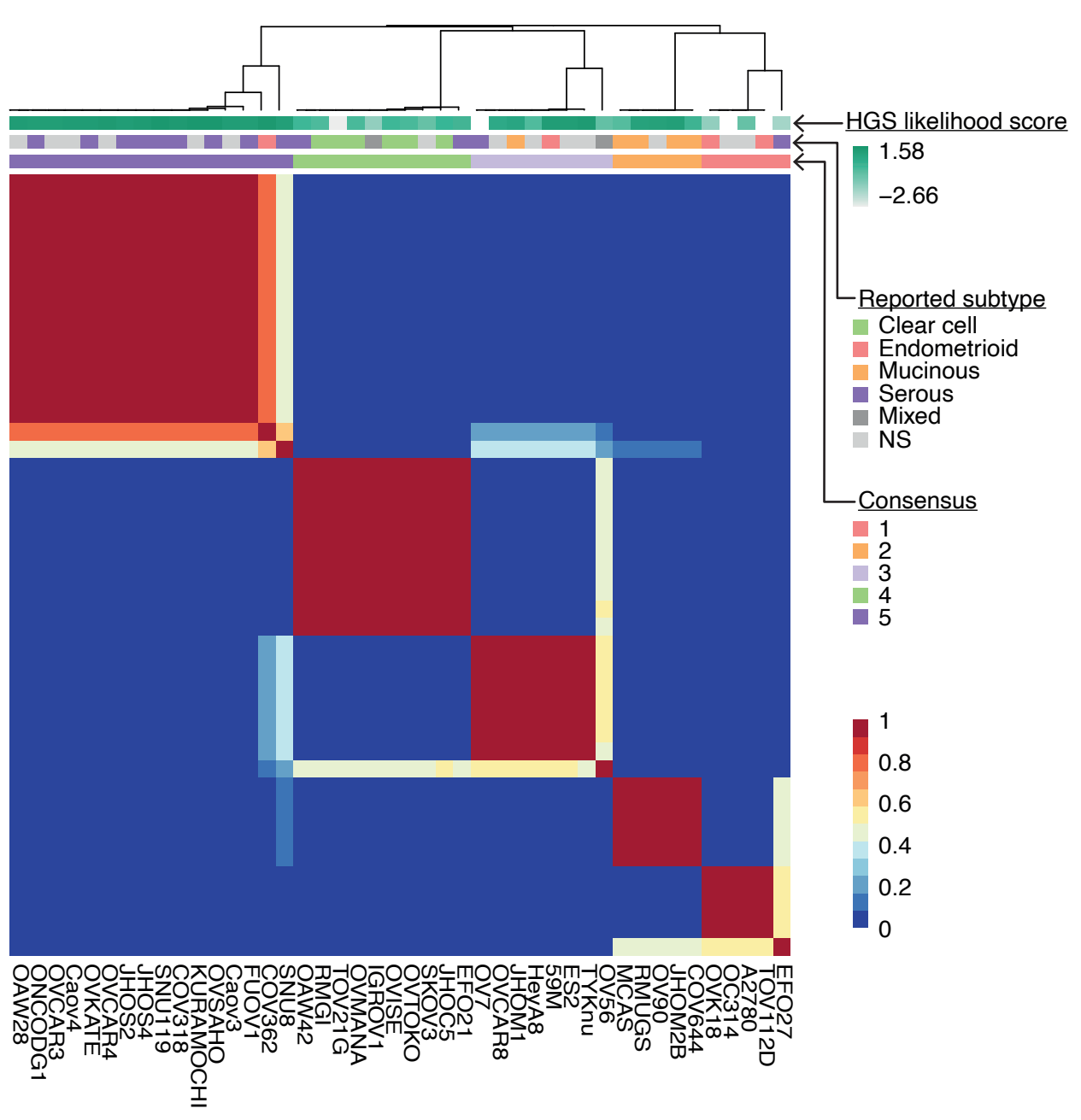

Figure 2 
bioRxiv preprint doi: https://doi.org/10.1101/2020.07.14.202457; this version posted July 15, 2020. The copyright holder for this preprint (which was not certified by peer review) is the author/funder. All rights reserved. No reuse allowed without permission.

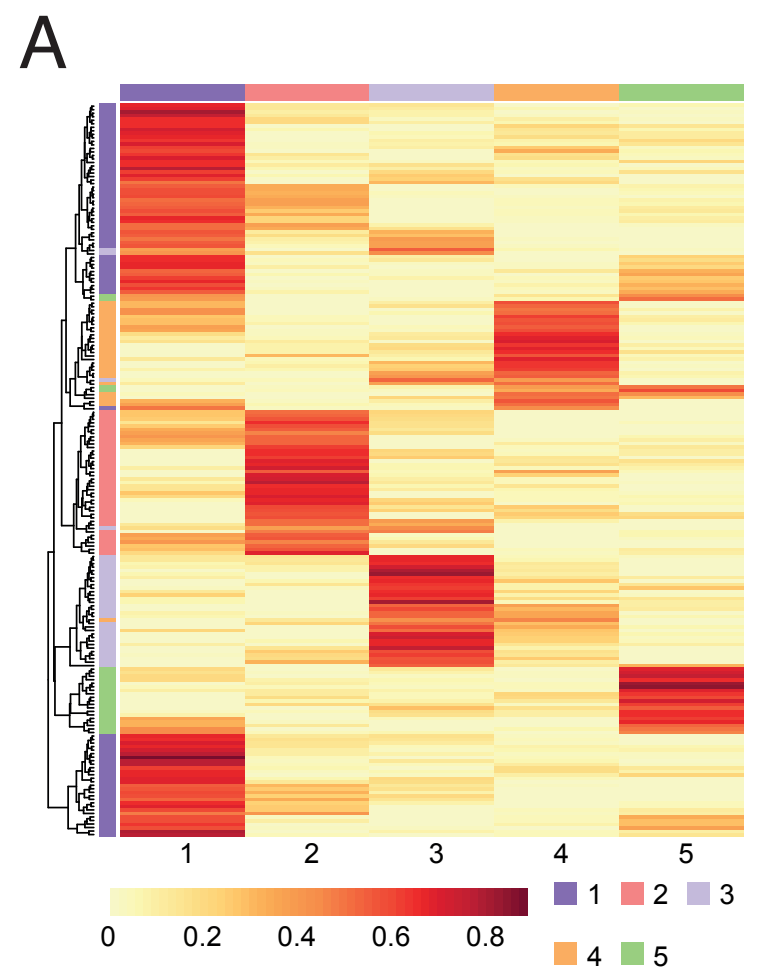

B

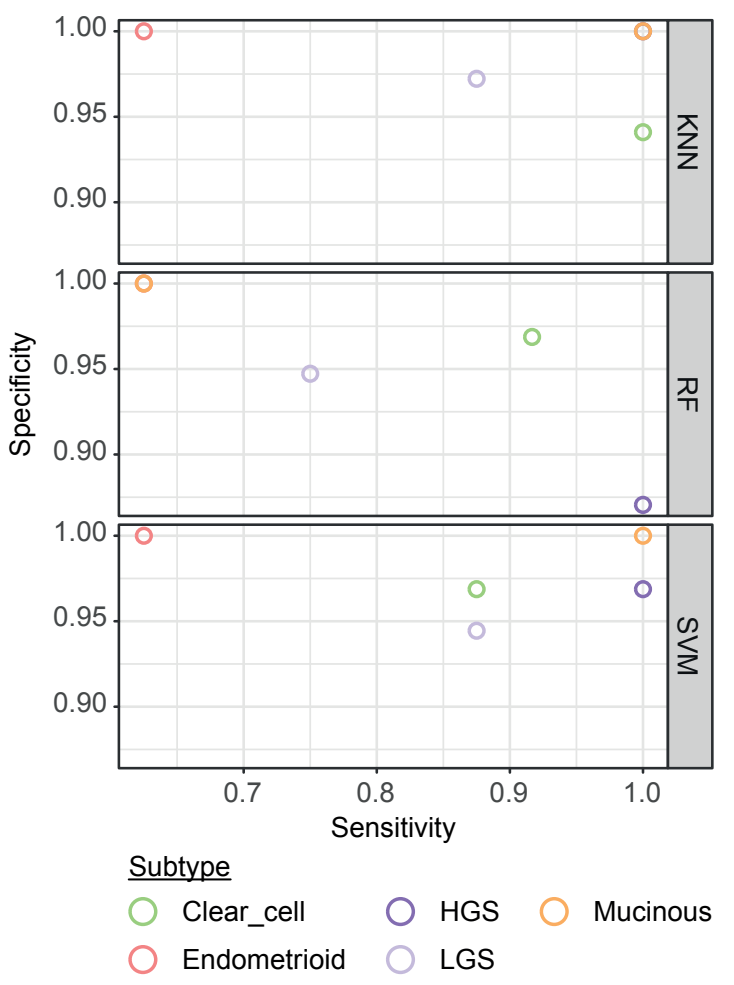


A

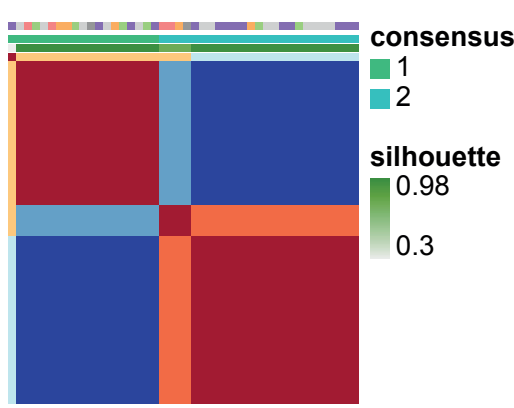

D

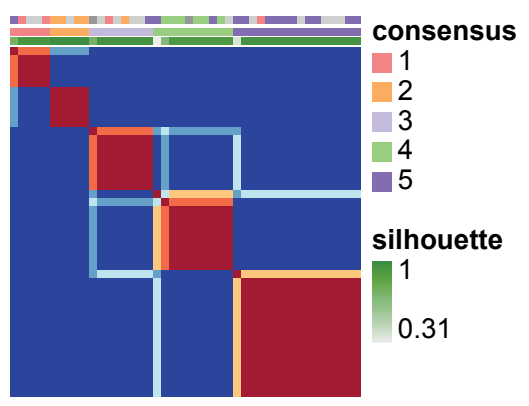

B

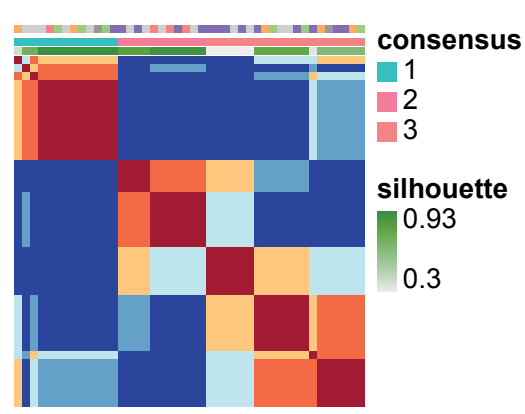

$E$

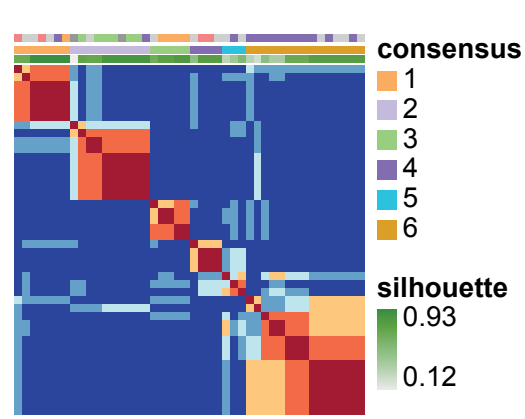

C

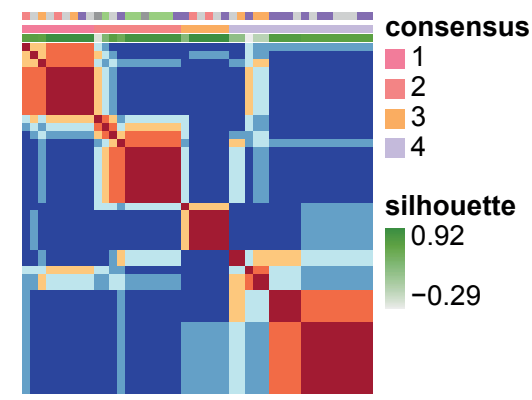

F

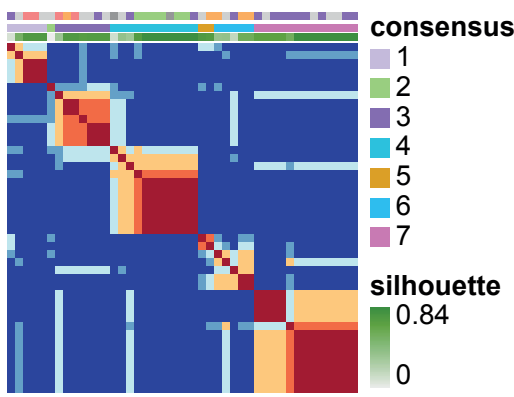

